\title{
Pour une lecture croisée des représentations de l'onde dans Bruges-la-Morte de Georges Rodenbach
}

\section{Ben Amor, Syrine}

Universitaire de la Manouba, syrine.ben.amor@hotmail.com

\begin{abstract}
Resumen
El agua es uno de los temas axiales de la obra de Georges Rodenbach. El tema común a lo largo de su producción escritural a lo largo de toda la obra es el agua que irriga la imaginación poética del escritor. ¿ Por qué Bruges-la-Morte ? Bruges-la-Morte es la novela de un viudo lloroso que ha elegido Brujas, ciudad de agua y canales, no para vivir en ella, sino para morir. Y es en Brujas, la Venecia de Flandes, donde continua buscando la cara de su esposa «sur d'autres visages ». Precisemos que el elemento del agua se presenta como la sustancia real del paisaje, así como del trabajo analógico en la novela.
\end{abstract}

Palabras clave : representaciones ; agua ; Bruges-la-Morte ; Georges Rodenbach.

\section{Résumé}

L'eau constitue l'une des thématiques axiales de l'œuvre de Georges Rodenbach. Thématique commune à sa production scripturale dans son étendue, l'eau irrigue l'imaginaire poétique de l'écrivain. Pourquoi Bruges-la-Morte ? Bruges-la-Morte est le roman d'un veuf éploré qui a choisi Bruges, ville d'eau et de canaux, non pour y vivre mais pour y mourir. Et, c'est à Bruges, la Venise flamande, que Hugues Viane s'acharne à chercher le visage de sa femme "sur d'autres visages ». Précisons que l'élément aquatique se présente comme la véritable substance aussi bien du paysage que du travail analogique entrepris dans le roman.

Mots-clés : représentations ; onde ; Bruges-la-Morte ; Georges Rodenbach.

\begin{abstract}
Water is one of the axial themes of the work of Georges Rodenbach. Common theme to its scriptural production in its extent, water irrigates the poetic imagination of the writer. Why Bruges-la-Morte ? Bruges-la-Morte is the novel of a tearful widower who has chosen Bruges, city of water and channels, not to live in it but to die in. And, it's in Bruges, the Flemish Venice, that HuguesViane persists in seeking the face of his wife" sur d'autres visages ». Let us specify that the element of water presents itself as the real substance of the landscape as well as of the analogical work undertaken in the novel.
\end{abstract}

Keywords : representations ; water ; Bruges-la-Morte ; Georges Rodenbach. 


\title{
Introduction
}

Dans cet article portant sur « les mots et les imaginaires de l'eau », nous avons opté pour un travail sur une œuvre phare de Georges Rodenbach. Nous avons uni dans ce travail l'étude et l'analyse des images de l'eau dans son roman-poème Bruges-la-Morte. Récurrentes et labiles sont les particularités des images poétiques et poétisantes de l'onde dans Brugesla-Morte de Georges Rodenbach. Plus intéressant nous semble le traitement des représentations de l'eau dans le roman. Sujettes à la modulation, à une distribution-redistribution sans cesse renouvelées, les images et les métaphores de l'onde fusent dans le roman. La présente lecture ambitionne de s'interroger sur la particularité des représentations de l'onde dans Bruges-la-Morte et de rendre compte de la profonde unité qui régit des images en apparence si contradictoires et disparates. Pour ce faire, nous proposons d'organiser notre propos en quatre moments. L'onde comme décor psychique est l'intitulé de la première partie. Nous passerons ensuite à la seconde partie que nous avons intitulé l'eau comme espace euphémique de la mort. S'agissant de la troisième partie, l'eau comme espace itératif, elle constitue une réflexion sur les rapports qu'entretiennent entre eux l'eau, l'œil et le miroir. La dernière partie de cet article porte sur le rapport eau/ texte, partie qui a pour titre : Eau et texte : lieux d'une configuration paradoxale.

\section{L'onde comme décor psychique}

L'auteur de Bruges-la-Morte réinvestit le topos romantique du paysage état d'âme pour en faire l'un des multiples «parallèle [s] » (Rodenbach, 1998 : 187) axiaux du roman. Mêlant leurs reflets, le personnage, la ville et ses eaux fusionnent. Comme paysage déclinant, la vieille cité Flamande, reflet de l'âme malade du veuf, et les eaux de ses canaux fonctionnent comme le parfait décalque de «ses humeurs noires » (Rodenbach, $1998: 181)$ et de «ses longs silences » (Rodenbach,1998: 181). Se plaçant lui-même au seuil de la société, Hugues Viane, l’infortuné, s’inscrit dans une marginalité voulue, voire fantasmée. Il espère ainsi perpétuer son image mythique de veuf inconsolable, d'en maintenir le rayonnement et de la fixer dans une ville faite essentiellement d'eau et de canaux. Pour un accroissement de la douleur, il opte ainsi pour une ville qui agonise car « son deuil exigeait un tel décor » (Rodenbach, 1998 : 30). La Flandre antique et mélancolique offre un décor propice à la vocation poétique de Hugues. En admirateur des romantiques français, le peintre de Bruges la convertit en une ville-état d'âme, une ville miroir. Bruges et ses eaux sont ainsi perçues comme un véritable paysage-miroir de sa détresse d'endeuillé. Il s'agit d'un décor psychique. En ce sens, l'élection d'une ville esthétiquement agonisante rend compte de l'agonie latente du personnage lui-même. La référence à l' "Aquarium mental » est incontournable. Dans ce poème, Rodenbach a figuré, métaphoriquement, la vie végétative du moi. Paul Gorceix traduit la similitude entre la ville et l'aquarium en ces termes : « Et Bruges se transforme en une sorte d'aquarium, immense et inquiétant, à l'intérieur duquel les êtres et les choses mènent une vie comparable à celle des "pensifs végétaux » de «l'Aquarium mental » (Gorceix, 1992 : 10). Citons le poème pour mieux saisir la parenté sous-jacente entre la ville et l'aquarium qui en devient la métaphore :

\author{
L'aquarium d'abord ne semble pas vivant, \\ Inhabité comme un miroir dans un couvent ; \\ Crépuscule où toujours se forme une brume ; \\ Il dort si pâlement qu'on le croirait posthume \\ Et que les reflets noirs qui viennent et s'en vont \\ Ne sont qu'ombre sans but sur un lit mortuaire \\ Et jeux furtifs de veilleuse sur le plafond (Rodenbach, $1896: 21$ ).
}

C'est ainsi que Bruges se transforme en un décor psychique et qu'une parfaite correspondance s'établit entre la ville et le veuf éploré. La métaphore de l'aquarium est ainsi prégnante. La descente dans les profondeurs abyssales du moi s'exprime chez Rodenbach par la référence à un matériau glauque et miroitant : celui de l'onde qui correspond, par extension, à celui de l'inconscient. Rappelons que HuguesViane choisit Bruges en amateur des correspondances non pour y vivre mais pour y mourir. Ainsi, « dans l'atmosphère muette des eaux et des rues inanimées, Hugues avait moins senti la souffrance de son cœur » (Rodenbach, 1998 : 69). Citons Bachelard qui nous dit: «contempler l'eau, c'est s'écouler, c'est se dissoudre, c'est mourir » (Bachelard, 1942 : 59). Et c'est principalement à travers l'eau de Bruges, cette «matière 
mélancolique » (Bachelard, 1998 : 75), que le personnage rodenbachien se dissout et meurt d'une mort métaphorique et foncièrement poétique. D’où la dimension lénitive, amollissante des eaux de Bruges. Et Rodenbach de nous dire : « Hugues avait senti, à l’origine, cette influence pâle et lénifiante de Bruges » (Rodenbach, 1998 : 194). La Flandre nordique se prête à merveille au projet de Hugues. C'est à travers l'eau de ses miroirs et de ses canaux mélancoliques qu'il s’adonne entièrement à sa douleur. L’élément aquatique se présente ainsi comme la véritable substance du paysage. Dans la Venise Flamande, cette ville d'eau où Hugues dissout sa peine, il peine à se dissoudre lui-même. Philippe Forest développe le rapport entre l'élément aquatique et l’intériorité psychique des personnages chez les symbolistes belges en ces termes :

[...] c'est l'intériorité de l'âme malade d'elle-même que les poètes belges vont s'attacher à explorer et à décrire. À travers les nombreuses images de milieux clos et aquatiques qu'ils dessinent, [...]. C'est là sans doute que se marque la spécificité envoûtante du symbolisme belge [...]. Se réfléchissant dans les paysages humides et comme morts que les cités et les plaines du nord peuvent proposer à une authentique imagination poétique, la poésie symboliste belge s'affirme comme une des plus impressionnantes explorations de l’intériorité la plus secrète de l’âme (Forest, 1989 : 95).

L'eau devient ainsi la représentation spatiale de l'état d’âme du personnage. L’onde n’est-elle pas cette « image matérielle particulièrement puissante et naturelle » (Bachelard, 1942 : 103), reflet éminemment poétique de son désespoir et de son deuil ? Comme l'affirme Gaston Bachelard, l'eau « dissout plus complètement. Elle nous aide à mourir totalement » (Bachelard, 1942 : 107). Plus loin, on lit : «L'eau meurt avec le mort dans sa substance. L'eau est alors un néant substantiel. On ne peut aller plus loin dans le désespoir. Pour certaines âmes, l'eau est la matière du désespoir » (Bachelard, 1942 : 108). En effet, les métaphores de la dissolution, dans leurs variantes, fusent dans le roman. Tel est, sans doute, la signification des verbes « s’ensabler » (Rodenbach, $1998: 133$ ) et « s'enliser » (Rodenbach, $1998: 133$ ). Si le personnage a choisi Bruges c’est pour « sentir ses dernières énergies imperceptiblement et sûrement s’ensabler, s'enliser sous cette poussière d'éternité » (Rodenbach, 1998 : 133). Comme variation métaphorique sur la mort de l'être, ces métaphores témoignent de la présence de la mort dans la vie. Le personnage rodenbachien, se soustrayant à l'agitation de la vie, opte pour la lente dissolution de soi. La métaphore de l'engouffrement en est, par extension, l'expression la plus aboutie. L'image de l'engouffrement et de la dissolution revêtent la même signification, elles deviennent ainsi des métaphores poétiques et poétisantes d'un long processus, celui de la mort dans et à travers les eaux de Bruges.

\section{L'eau comme espace euphémique de la mort}

Comme espace de la mort, les eaux des canaux de Bruges sont représentées comme le prolongement spatial, métaphorique et métonymique du corps gisant de la défunte épouse, de ses cheveux en tresse et de la ville. Les eaux et les canaux de Bruges sont systématiquement associés à l’immobilité et au silence. Il est en effet question des "solitaires canaux » (Rodenbach, 1998 : 54) et de « l'atmosphère muette des eaux » (Rodenbach, 1998 : 69) de Bruges. L'image matricielle des eaux mortes rejoint celle de la ville gisante et de la défunte par la référence à une horizontalité symbolique. Nous tenons à citer quelques fragments du texte qui sont représentatifs de la dimension mortifère, symbolique et métonymique des eaux de Bruges :

- De Bruges-la-Morte (la ville) et de ses canaux, est dit : « C’était Bruges-la-Morte, elle-même mise au tombeau de ses quais de pierre, avec les artères froidies de ses canaux, quand avait cessé d’y battre la grande pulsation de la mer » (Rodenbach Georges, 1998 : p. 69-70).

- De la défunte est dit : « [...] la jeune femme était morte, au seuil de la trentaine, seulement alitée quelques semaines, vite étendue sur ce lit du dernier jour, où il la revoyait à jamais : fanée et blanche comme la cire l'éclairant [...] ».

- À propos de la tresse de « la Regrettée », Rodenbach nous dit : « c’était la chose même de la morte, qui avait échappé à la tombe pour dormir d'un meilleur sommeil dans ce cercueil de verre [...] » (Rodenbach, 1998 : 141).

Les fragments descriptifs précités rendent compte de la parenté sous-jacente que Rodenbach met en place entre les eaux de Bruges, la femme et la ville. Il est question d'une horizontalité mortifère, celle de la mort. Les eaux et la ville sont ainsi représentées comme un prolongement, une ramification du corps gisant de la défunte. La ville, à travers ces canaux, 
reflète l'image de la défunte sur son « lit du dernier jour » (Rodenbach, 1998 : 53). Par un jeu de correspondances, Rodenbach inscrit le visage de la défunte dans le paysage essentiellement liquide de la ville. La femme et la ville finissent par s'unir et fusionner par l'effet d’un destin unique de sorte que, pour Hugues, « tout s'unifiait en une destinée pareille » (Rodenbach, 1998 : 68). Il y a acheminement vers l'expression d'une fusion totale, voire d'une incorporation. Le sémantisme du verbe «s'unifier » en est révélateur. Avec Rodenbach, la ville et ses eaux deviennent un réceptacle de mort, non de vie : la ville porte en son sein, au fond de ses eaux, le corps de la défunte. « Il l'avait [en effet] mieux revue, mieux entendue ; retrouvant au fil des canaux son visage d’Ophélie en allée, écoutant sa voix dans la chanson grêle et lointaine des carillons » (Rodenbach, 1998 : 69). Ici, nous retenons l'image focale de l'incorporation. L'intérêt poétique de l'image est primordial. La ville, mater dolorosa, semble envelopper la morte, la porter en son sein de sorte que leurs deux images se confondent, maternellement, en un seul et unique tableau. La mort dans les eaux n'est-elle pas, selon l'expression de Bachelard, « la plus maternelle des morts » (Bachelard, 1942 : 89)? Georges Rodenbach, met en place un large réseau associatif, une sorte de jeu de miroir où les images sont, intentionnellement, miroitantes. Les images de la femme, de la tresse, de la ville et des eaux de Bruges nous ramènent au modèle premier de l'eau. Le miroitement, n'estil pas le propre des eaux? C'est ainsi que ces mêmes images deviennent interchangeables: l'eau est certes la représentation métonymique et métaphorique de la ville mais c'est aussi une représentation de la femme morte. L'eau renvoie à la tresse, par extension, à la femme morte, et, pour clore la liste, à la ville morte, Bruges-la-Morte. Afin de signifier pleinement la mort des eaux de Bruges, de la défunte et de la ville elle-même, l'auteur opte pour un mode descriptif particulier. Le matériau descriptif employé nous renseigne sur la tentation sculpturale de ses descriptions. Par les mots, le langage, Rodenbach écrit, peint et sculpte. Et c'est en immobilisant les images qu'il en arrive à les fixer pour attester, paradoxalement, et de leur mort (puisqu'il les fixe) et de leur persistance (en les affranchissant des règles de la finitude imposées par la mort) en les présentant tantôt comme des tableaux tantôt comme des sculptures. Nous proposons d'examiner le passage descriptif suivant : «C’était Bruges-la-Morte, elle-même mise au tombeau de ses quais de pierre avec les artères froidies de ses canaux, quand avait cessé d’y battre la grande pulsation de la mer » (Rodenbach, 1998 : 69-70). Rodenbach sollicite ici l'image de la pierre. La pierre comme métonymie de la statue ou de la sculpture est omniprésente dans Bruges-la-Morte. Si le texte, en tant que tissu lexical, exprime une tentation vers la figuration sculpturale, c'est principalement en faveur du rapport qu'il entretient avec la thématique centrale de la mort. Le figement devient la traduction de la mise en tombeau. La référence à la pierre est ainsi éloquente. C’est en faveur de sa rigidité et de l’immobilité qui lui est inhérente que l’auteur ne cesse de l'évoquer. Dans sa description de Bruges, Rodenbach représente la ville comme «rigide et [...] emmaillotée dans les mille bandelettes de ses canaux » (Rodenbach, 1998 : 125). Comme métonymie du tombeau, la pierre traduit la mise en tombeau de toute la ville. L'auteur explicite l'image du tombeau en ces termes : « [...] et il marchait, consolé, à travers son silence, comme si Bruges avait surgit de son tombeau [...]» (Rodenbach, 1998 : 125). La ville de Bruges, ville d'eau, devient ainsi sous la plume de Rodenbach une cité pétrifiée aux eaux mortes, car figées. L’auteur de Bruges-la-Morte, établit un parallèle entre l’immobilité de la mort et celle des entités représentées comme mortes du roman : les eaux, la ville et la femme.

\section{L'eau comme espace itératif : eau, œil, miroir}

L'eau se présente comme un monde en creux qui invite le regard à y plonger pour saisir l'image qu'elle abrite. Elle constitue à elle seule un monde à part où l'image erre mais ne se perd pas. Elle y est ancrée, elle s'enracine. L'eau comme espace itératif constitue ainsi aussi bien une surface réfléchissante qu’un espace aux propriétés enchâssantes. L’eau rodenbachienne rejoint le miroir et l'œil compte tenu d'un mode de fonctionnement qui leur est commun. Il y a ainsi dans Bruges-la-Morte (la ville et le roman) une surabondance de surfaces réfléchissantes et enchâssantes qui se présentent dans leur diversité. Ces variantes de l'eau-miroir embrayent un texte où convergent reflets et images. Leur principale vertu est de contribuer à l'emprisonnement/protection des images et de la trace de la défunte. Ainsi le reflet dans Bruges-la-Morte est loin de se réduire à ce que le regard perçoit seulement en surface. Quels sont donc les différents visages de l'eaumiroir enfouis dans un texte lui-même construit en miroirs ? Quels rapports entretiennent entre eux l'eau, l'œil et le miroir ? Dans Bruges-la-Morte, l'onde est systématiquement et explicitement associée à l'œil et au miroir. L'exploration des associations syntagmatiques entreprises dans le roman permet de relever cette parenté. Il est question des eaux stagnantes de Bruges et des «yeux stagnants » du personnage. Chez l'auteur, l'eau et l'œil deviennent équivalents, interchangeables. Il y a synonymie. À l'œil sont attribuées les caractéristiques de l’onde et vice versa. À l'instar de l'eau, 
l'œil et le miroir «emmagasinent» et captent l'image. Citons ces extraits qui rendent compte de cette capacité d’absorption de l'image :

Il cherchait dans ce visage la figure de la morte. Pendant de longues minutes, il la regardait, avec une joie douloureuse, emmagasinant ses lèvres, ses cheveux, son teint, les décalquant au fil de ses yeux stagnants... Élan, extase du puits qu’on croyait mort et où s'enchâsse une présence. L'eau n’est plus nue ; le miroir vit (Rodenbach, 1998 : 106) !

Plus loin, «Quand il prenait dans ses mains la tête de Jane, l’approchait de lui, c'était pour regarder ses yeux, pour y chercher quelque chose qu'il avait vu dans d'autres : une nuance, un reflet, des perles, une flore dont la racine est dans l'âme - et qui y flottaient aussi peut-être » (Rodenbach, 1998 : 112). L'exploration des associations syntagmatiques permet de relever la parenté synonymique entre des verbes qui deviennent interchangeables, presque identiques tels que : « cherch [er], emmagasin [er], décalqu [er] » (Rodenbach : 106) et « s’enchasse [r] » (Rodenbach, 1998 : 106). L’œil et l'eau chez l'auteur de Bruges-la-Morte sont dotés d'une spatialité fantasmatique. Ils acquièrent une profondeur qui permet à l'image de s'y incorporer, d'y dormir selon une expression chère à Rodenbach. L'œil qui est le foyer du regard devient l'habitat et le réceptacle de l'image qui s’y loge dans un infini abandon. Il est capital de signaler que le mode de fonctionnement du regard dans Bruges-la-Morte nous renvoie à celui si particulier et presque magique du miroir et de l'eau du fait de sa capacité d'intériorisation. À l'instar de l'eau, l'œil devient le réceptacle de l'image. Le reflet et l'image subissent ainsi l'acte d’intériorisation que dicte le désir de possession de Hugues Viane. Nous avons lieu de voir dans la configuration spatiale de l'eau et de l'œil que l'intériorisation se meut en absorption. Citons un commentaire du narrateur qui traduit l'effet que produit la rencontre de Jane sur Hugues Viane et le processus qu'elle déclenche chez lui : « Son œil avait emmagasiné le cher visage une seconde fois » (Rodenbach, 1998 : 82). Le sémantisme du verbe " emmagasiner » est à ce titre significatif. Aussi, à la lumière de la logique rodenbachienne, le verbe traduit la soif de l'appropriation et le désir de dévoration. Il est également intéressant de remarquer que les verbes « emmagasiner, s’enchâsser et décalquer » sont attribués à toutes les surfaces réfléchissantes dans Bruges-la-Morte. Paul Edwards, dans son article « The photograph in Georges Rodenbach's Bruges-la-Morte », cite l'un des poèmes de Rodenbach qui s’intitule « Voyage dans les yeux » et qui nous renseigne sur cet espace fascinant qu'est l'œil :

Car tout s'y fige, y dure, et tout s’y perpétue : Désirs, mouvements d’âme, instant, décor, Tout ce qui fut, rien qu'un moment, y flotte encore; (...) Et l'on voit, dans les yeux (...) d'anciens amours mirés comme de grands tombeaux (Rodenbach, 1896 : 146).

Signalons par ailleurs qu'il y a chez Rodenbach, aussi bien dans Bruges-la-Morte qu'ailleurs, une association analogique entre l'œil et l'eau. Allant dans ce sens, Patrick Laude affirme que : « la plasticité multiforme de l'eau - comme celle de “l'eau” des yeux -, assimilée à toute réalité de "surface », de facticité et de jeu, implique une des orientations majeures de l'univers imaginaire rodenbachien: le sens de l'illusion » (Laude, 1999 : 40). Ailleurs, précisément dans son poème « Le Voyage dans les yeux », Rodenbach n’hésite pas à reprendre et développer cette idée en décrivant l'œil en ces termes : «L'œil est un glauque aquarium d'eau somnolente », il est aussi «cet écran de cristal » sur lequel viennent s’inscrire les spectacles formels dans toute leur diversité » (Laude, 1999 : 44). Dans Bruges-la-Morte, Rodenbach tisse des équivalences semblables en qualifiant les yeux de Jane de «stagnants » (Laude, 1999 : 254). Il opère ainsi un rapprochement entre l'œil et l'eau qui deviennent les instruments du dédoublement. Dans Bruges-la-Morte, les canaux et les eaux mortes de la ville sont associés à l’absence de la femme que le personnage revoit au fil des canaux de Bruges. L'eau et les rivières occupent dès lors une grande place dans l'univers rodenbachien et fonctionnent comme des miroirs réfléchissant les images aussi bien de la ville que de la défunte. Les propos du narrateur nous renseignent sur la similitude établie entre l'eau et le miroir : des miroirs est dit « qu’on s’y voyait comme dans des pièces d'eau » (Rodenbach, 1997 : 24). L'eau et le miroir deviennent ainsi interchangeables. Dans le roman, il y a développement de toute une rhétorique du miroir. Le motif du miroir, comme modulation sur la thématique de l’onde, constitue ainsi un thème clef chez Rodenbach. Il est, à l'instar de l'eau et de l'œil, le lieu du redoublement et de la multiplication de la perspective. Il se présente comme garant de la persistance de l’image de la défunte. Il permet de conserver sa trace. Il est ainsi doté d'une profondeur et 
d'une épaisseur. Outre sa fonction essentielle, celle qui consiste à refléter l'image, lui est attribué, symboliquement, un pouvoir d'appropriation de l'image qu'il est à même d'intérioriser et d'éterniser. Il est, dans la logique rodenbachienne, à l'instar de l'onde et de l'eau en mesure de préfigurer l'absence, de retenir l'image. Il arrive ainsi à fixer l'image évanescente de la morte, à se l'approprier en l'enfouissant. Rodenbach nous dit : « Et dans les miroirs, il semblait qu'avec prudence il fallût en frôler d'éponges et de linges la surface claire pour ne pas effacer son visage endormi au fond » (Rodenbach, 1998 : 58). Le miroir rodenbachien conjugue la réflexion et l'absorption. Il est transparence et profondeur. Rodenbach élabore une mythologie, peut-être même une mystique du miroir qui transcende son statut de simple objet. Comme lieu de la redondance, comme « opérateur de mise en abîme » (Bertrand, 1990 : 50), le miroir devient aussi et surtout l'habitat de la mort. Le miroir communique avec la morte. Entre elle et lui, il y a connivence et sympathie. Il retient son visage. Il le porte en creux comme les canaux de Bruges retiennent, en le reflétant, « son visage d’Ophélie en allée » (Rodenbach Georges : 69). N’est-il pas, à l’image des eaux de Bruges, le lieu privilégié des endeuillés où « le visage des morts subsiste » (Rodenbach, 1998 : 254) ? Si nous avons décidé de réfléchir sur la particularité des surfaces réfléchissantes dans Bruges-la-Morte, c’est pour démontrer comment leur configuration obéit au modèle premier de l'eau. L'œil, l'eau et le miroir deviennent des « opérateur [s] de mise en abîme » (Bertrand, 1990 : 66). À l'instar du miroir et de l'œil qui conservent, jalousement, l'image de la disparue, l'eau garde, maternellement, son reflet. Mais n'est-ce pas d'une aporie qu'il s'agit car au moment même où Rodenbach qualifie de mortes les eaux de Bruges, en en faisant le réceptacle des images mortes et de la ville et de la femme, il en vient à doter ces mêmes eaux mortes de vie ?

\section{Eau et texte : lieux d'une configuration paradoxale}

L'analyse des surfaces réfléchissantes nous a permis de démontrer que ces mêmes espaces où le reflet se fixe et l'image se loge ne sont que des tombeaux ouverts. Chez Rodenbach, les eaux-miroirs, comme l'a si bien dit Aurore Boraczek, sont « dotés de mémoire » (Bertrand, 1990 : 66). L’eau comme espace paradoxal, se présente comme la matérialisation d'un état de présence/absence. L'eau rodenbachienne, en portant les images mortes de la femme et de la ville, les présentifie pour en faire une persistance. Dans les eaux de Bruges-la-Morte «s'enchâsse [toujours ou presque] une présence » (Rodenbach, 1998 : 106). Plus intéressante nous semble la capacité de l'eau rodenbachienne à figurer l'absente et, par extension, l'absence. L'eau des canaux de Bruges constitue un espace frontalier, un entre-deux dynamique où deux univers se croisent : la mort et la vie. Par ailleurs, le choix de la préposition « dans » n’est pas anodin. C’est la préposition qui sied le plus et d'une manière paradoxale aussi bien à la représentation de la mort (à travers l'image de l'enterrement qu'elle véhicule), qu’à l'expression d’un semblant de victoire sur la mort. En effet, "être dans » c'est certes être en quelque sorte inhumé, enterré, mais c'est également continuer à être, à exister. Chez Rodenbach, paradoxalement, seuls les morts ont droit au reflet. Le modèle vivant est banni parce que l'eau-miroir ne peut être que le reflet des images statiques, celles de la mort. L'eau, l'œil et le miroir sont, dans Bruges-la-Morte, les gardiens de l'authenticité du souvenir. Il s'agit donc d'être dans l'eau-miroir, au fond des eaux de Bruges ou de ne pas être totalement. Le texte et les eaux de Bruges ont ceci en commun qu'ils sont aptes à fixer les images du passé et à figurer l'absence. Par ailleurs, Bruges-laMorte, le roman, serait à lire comme une tentative de figement, de fixation d'une vieille ville que menace l'effritement de l'oubli. Rappelons que, selon la logique réflexive des lieux clos dans le roman, les eaux de Bruges permettent de reproduire les images de la vieille cité flamande et de multiplier ses reflets. L'eau, cet espace paradoxal qui fait conjoindre la mort et la vie, est le reflet d'un texte qui est lui-même l'habitat de l'absente et de l'absence. L'expérience de l'absence aboutit, toutefois, à la présence du texte. D’où la dialectique de la mort et de la vie qui traverse et le roman de Georges Rodenbach et les eaux des canaux de Bruges-la-Morte.

\section{Conclusion}

Le choix d'une ville d'eau et de canaux est une entrée dans les profondeurs de l'inconscient de l'homme. C'est un univers glauque, fluctuant et instable qui traduit les tourments et les incertitudes du moi. L'espace, dans sa liquidité, devient le miroir où s’agitent les images antérieures d'un passé transposé sur la scène du langage. Le personnage rodenbachien évolue dans un paysage liquide et humide aux contours raréfiés et incertains qui rappellent l'univers onirique. À l'instar de Bruges, ville miroitante faite d'eau et de canaux, le texte est un espace flottant et, partant, instable. Les eaux de Bruges sont pour ainsi dire le garant de la poéticité du roman. L’eau irrigue l’imaginaire poétique de l’écrivain. La labilité qui caractérise les manifestations et les représentations de l'onde dans Bruges-la-Morte nous rappelle que le roman est 
l'œuvre d'un poète. Si l'eau est l'espace de l'imagination, le roman, quant à lui, est l'espace de l'illusion. Entouré du blanc typographique, le texte se présente comme la revendication d'une rupture avec le réel, ou, d'un détachement du réel. Il y a création d'une atmosphère de flottement qui n'est pas sans rappeler le miroitement des eaux des canaux de Bruges. Le texte, de par le blanc qui l'entoure, semble flotter sur la page blanche. Le blanc typographique est ainsi, à l'image de l'eau, l'espace de l'imagination, le lieu de l'imaginaire. Le texte semble emprunter ses thématiques, sa construction et sa syntaxe à l'onde ou, simplement, à l'eau. En bref, si l'eau capte le reflet en l'enfouissant, le texte, quant à lui, capture ce même reflet par les mots, le langage. Les mots couchés sur la page deviennent chez Rodenbach des gisants. Comme le dit si bien Bachelard, l'eau « donne sa matière à l'imagination créatrice » (Bachelard, 1942 : 211 ). L'eau offre sa substance, sa liquidité au texte. La coulée phrastique n'est pas sans rappeler celle de l'eau. Et c'est ici le lieu de rappeler la nécessité d'attirer l'attention sur l'homophonie eau/mot et d'en souligner toute sa pertinence. L'eau génère les mots.

\section{Références bibliographiques}

BACHELARD, Gaston (1942). L'Eau et les Rêves : essai sur l'imagination de la matière. Paris : José Corti.

BERTRAND, Jean-Pierre (1990). Le Monde de Rodenbach. Bruxelles : Labor.

EDWARDS, Paul (1892). «The photograph in Georges Rodenbach’s Bruges-la-Morte » dans Journal of European Studies, World History in Context, March 2000.

FOREST, Philippe (1942). Le symbolisme ou naissance de la poésie moderne. Paris : Pierre Bordas et Fils.

GoRCEIX, Paul (1992). Réalités flamandes et symbolisme fantastique Bruges-la-Morte et Le Carillonneur. Paris : Archives des Lettres Modernes.

LAUDE, Patrick (1999). Rodenbach Les Décors De Silence. Bruxelles : Labor.

RoDenBACH, Georges (1997). Le Rouet des brumes. Atlantica : Séguier.

RodenBACH, Georges (1998). Bruges-la-Morte. Paris : Garnier Flammarion.

RoDEnBACH, Georges (1896). Les Vies Encloses. Paris: Fasquelle. 